\title{
Role and mechanism of action of LAPTM4B in EGFR-mediated autophagy (Review)
}

\author{
XIAOKUN JI, HUA MA and YUN DU \\ Department of Cytology, The Fourth Hospital of Hebei Medical University, Shijiazhuang, Hebei 050011, P.R. China
}

Received November 12, 2021; Accepted January 17, 2022

DOI: $10.3892 / 01.2022 .13229$

\begin{abstract}
LAPTM4B is upregulated in the majority of types of cancer and associated with cancer cell proliferation, survival and drug resistance, as well as poor patient prognosis. LAPTM4B knockdown inhibits autophagosome maturation in the context of metabolic stress. Autophagy is a homeostatic process that degrades and recycles intracellular components in response to metabolic stress. The function of autophagy is dual, as this process can either have a tumor suppressor or an oncogenic role. EGFR serves an important role in determining the tumor-suppressive or oncogenic roles of autophagy. EGFR family members regulate autophagy through various signaling pathways, including PI3K/AKT signaling. Notably, LAPTM4B also promotes cancer cell proliferation via the PI3K/AKT signaling pathway. In addition, LAPTM4B can enhance and prolong EGFR signal transduction by blocking active EGFR intraluminal sorting and lysosomal degradation. Thus, LAPTM4B may be associated with autophagy through EGFR signaling. The present review proposed that LAPTM4B participates in regulating autophagy through the EGFR pathway.
\end{abstract}

\section{Contents}

1. Introduction

2. LAPTM4B

3. The dual role of autophagy in human malignancies

4. EGFR-mediated autophagy pathways

5. LAPTM4B and EGFR

6. Conclusions and future perspectives

Correspondence to: Professor Yun Du, Department of Cytology, The Fourth Hospital of Hebei Medical University, 12 Jiankang Road, Shijiazhuang, Hebei 050011, P.R. China

E-mail: yydd40ss@aliyun.com

Key words: LAPTM4B, autophagy, EGFR

\section{Introduction}

Autophagy is an evolutionarily conserved lysosome-mediated recycling process that is essential for the maintenance of cellular homeostasis and nutrient recycling $(1,2)$. Autophagy is characterized by the formation of autophagosomes, which are vesicles with a lipid bilayer (1-3). Cytoplasmic constituents are engulfed into the autophagosome, which is delivered to the lysosome for degradation or nutrient recycling $(1,2)$. Autophagosome formation, elongation, maturation and degradation are regulated in a time-dependent manner by autophagy-related genes (ATGs), such as Beclin 1, VPS34, ATG14, VPS15 and LC3 $(1,3)$.

EGFR is a member of the ErbB tyrosine kinase transmembrane receptor family that is activated following binding to its ligand, EGF (4). EGFR signaling pathways serve important roles in cell survival, growth, proliferation and differentiation $(5,6)$. EGFR can regulate epithelial tissue development and homeostasis under physiological conditions; however, it can also become a driver of tumorigenesis (7). In addition, EGFR also regulates the response of cancer cells to metabolic stress (6). Various types of cancer, such as non-small cell lung cancer, and head and neck carcinomas, are associated with EGFR mutations or upregulation (8). Targeted therapy for EGFR sensitive mutations such as 19 deletion, L858R and G719X with EGFR-tyrosine kinase inhibitors (TKIs) has become an important therapeutic method $(8,9)$. However, drug resistance often develops following EGFR-TKI therapy (10). It has been demonstrated that autophagy serves an important role in drug resistance of EGFR-TKIs $(11,12)$. A recent study has shown that EGFR signaling suppresses autophagy (13).

The LAPTM4B gene is located on chromosome 8q22.1 and was first identified in human hepatocellular carcinoma (HCC). LAPTM4B is a novel tetratransmembrane protein primarily localized in the late endosomes and lysosomes (14). Notably, endosomes may provide a membrane source for autophagosome (15). Lysosomes are essential for autophagosome maturation and degradation (3). It is also reported that LAPTM4B is required for autophagy initiation (16). Furthermore, LAPTM4B knockdown inhibits autophagosome maturation and autophagic flux in the context of a metabolic stress microenvironment (16-18). However, the role and mechanism of action of LAPTM4B in autophagy remains unclear. The present review proposes that LAPTM4B participates in regulating autophagy through the EGFR pathway. 


\section{LAPTM4B}

The LAPTM4B gene is mapped to chromosome 8q22.1 (19). The two alleles of LAPTM4B gene, AY219176 and AY219177, encode 35- and 40-kDa proteins, respectively (19). Previous studies have demonstrated that polymorphisms in the LAPTM4B gene were associated with tumorigenesis, tumor proliferation and metastasis (20-24).

LAPTM4B is upregulated in several types of cancer and associated with cancer cell proliferation, survival, drug resistance and poor prognosis (19). For instance, the LAPTM4B-35 protein encoded by the AY219176 allele of LAPTM4B is upregulated in HCC, lung cancer, extrahepatic cholangiocarcinoma, gallbladder cancer and breast cancer (22-25). The LAPTM4B-35 protein promotes proliferation and chemotherapy resistance of carcinoma cells through the AKT signaling pathway and contributes to cell migration, invasion and metastasis via its proline-rich domain (PPRP motif), which can interact with SH3 domain-containing signaling molecules that are involved in several signaling pathways (25-27). A previous meta-analysis demonstrated that the AY219177 allele of LAPTM4B, encoding the $40-\mathrm{kDa}$ protein, is a high-risk factor for cancer (24). LAPTM4B promotes chemotherapy resistance by increasing drug efflux and decreasing drug nuclear localization and drug-induced DNA damage in vitro and in vivo $(18,27,28)$. LAPTM4B upregulation is associated with tumorigenesis, drug resistance and poor prognosis in hepatocellular, gallbladder, extrahepatic cholangiocarcinoma, ovarian, gastric and colon carcinoma $(26,29-34)$ through autophagy (35). LAPTM4B silencing using short interfering (si)RNA inhibits autophagic flux, whereas its overexpression rescues cell autophagy flux in the context of metabolic stress $(16,18)$. LAPTM4B can regulate autophagy by activating ATG3 transcription, which has been predicted using Gene Ontology analysis and animal experiments (17). However, the role of LAPTM4B in autophagy and the underlying mechanism remains to be elucidated.

\section{The dual role of autophagy in human malignancies}

Autophagy is a conserved lysosome-mediated type-II programmed cell death, which is specifically regulated by ATGs. Basal autophagy is essential for homeostasis and can be upregulated in response to metabolic stresses as a cell survival mechanism. Excess autophagy results in cell death by catabolizing essential cellular components $(36,37)$.

Autophagy has a dual function, as it can act both as a tumor suppressor and promoter. In normal cells, homeostatic autophagy can keep cells from malignant transformation by degrading intracellular toxic components. Nevertheless, autophagy can counteract hypoxia, nutrient starvation and exposure to chemotherapy, allowing cancer cells to adapt to these stress conditions and promoting their survival. Autophagy also serves an important role in chemoresistance in osteosarcoma, as well as ovarian and lung carcinoma (38-44). A series of clinical trials have investigated the role of autophagy inhibitors, such as hydroxychloroquine (HCQ) or chloroquine (CQ), and autophagy inducers, including rapamycin, in tumor therapy (45-49). These results showed that HCQ or CQ inhibits autophagy, resulting in increased tumor shrinkage. HCQ (the only clinically-approved autophagy inhibitor) with an improved toxicity profile compared with CQ has been studied in several phase I/II clinical trials alone or in combination with other chemotherapeutic regimens such as cisplatin (35). However, rapamycin as an autophagy inducer also shows cytotoxic effects in tumor targeted therapy (35). Recent studies show that ATGs are involved in mediating resistance to chemotherapy and may represent potential therapeutic targets (50-54). EGFR serves an important role in determining whether autophagy will serve a tumor-suppressive or an oncogenic role (6).

\section{EGFR-mediated autophagy pathways}

EGFR is a transmembrane tyrosine kinase receptor that can regulate DNA synthesis and cell proliferation and serves critical roles both in physiological conditions and in cancer $(8,55)$. Various types of cancer, such as non-small cell lung cancer, and head and neck carcinomas, are associated with EGFR mutations or upregulation (8). Notably, it has been reported that the activation of EGFR by its ligand, EGF, inhibits autophagy $(13,16)$.

EGFR regulates autophagy through the activation of the PI3K/AKT/mTOR (56), EGFR-RAS (57-59), EGFR-Beclin 1 (13) and EGFR-STAT3 signaling pathways (Fig. 1). PI3K, AKT and mTOR, which are downstream molecules of the EGFR signaling pathway, negatively regulate autophagy through inhibition of the ULK1 complex (6). Activated EGFR phosphorylates PI3K, AKT and TSC1, which activates mTOR and leads to the formation of the mTOR complex (mTORC1 or mTORC2). The multiprotein complex mTORC1 negatively regulates autophagy through the phosphorylation of ULK1, which interferes with the interaction between ULK1 and AMPK thereby preventing the formation of ULK1, FIP200 and ATG13 complexes (5,60-62). UVRAG, which regulates autophagosome maturation by binding to HOPS (homotypic fusion and vacuole protein sorting) complex, can be phosphorylated by mTORC1. RUBICON antagonizes the HOPS complex and interacts with UVRAG to inhibit UVRAG-mediated autophagosome maturation (63). EGFR family members activate the RAS/MAPK pathway, which activates RAF. Activated RAF activates MEK1/2 and ERK1/2 through the phosphorylation of their kinase domains. The RAS/RAF-1/MEK1/2/ERK pathway promotes autophagy by blocking the interaction between Bcl-2/Mcl1 and Beclin $1(58,62,64)$. In addition, it has been reported that the RAS signaling pathway may promote autophagy by upregulating ATG5 and ATG7 expression $(57,65)$. EGFR suppresses autophagy by interacting with the Bcl-2-homology-3 and evolutionarily conserved domain of Beclin 1, which results in multisite tyrosine phosphorylation of Beclin 1 on residues Y229, Y233, and Y352 and increases binding to the negative regulators, $\mathrm{Bcl}-2$ and RUBICON, but decreases binding to the VPS34 lipid kinase. In addition, EGFR signaling upregulates the expression of the anti-autophagic protein Bcl-2 through the STAT3 pathway. Activated STAT3 then translocates to the nucleus to upregulate of $\mathrm{Bcl}-2$ expression $(66,67)$. Bcl-2 inhibits autophagy by binding to Beclin 1 through its BH3 domain (68). Cytoplasmic STAT3 interacts with PKR through its SH2 domain, thereby preventing eIF2 $\alpha$ hyperphosphorylation. Consequently, autophagy is inhibited by preventing LC3b and ATG5 cascading initiation $(69,70)$. 


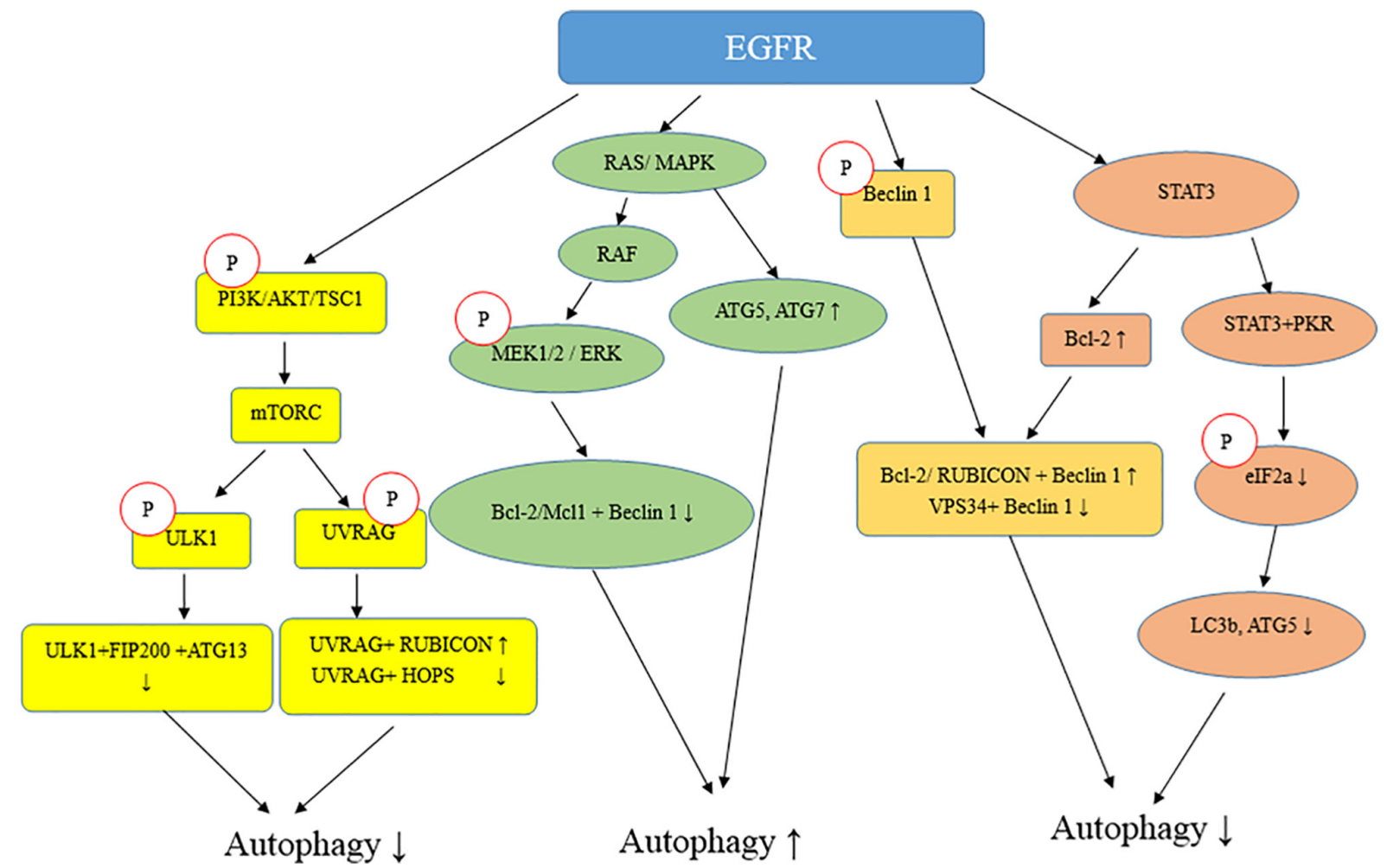

Figure 1. The EGFR signaling pathway is involved in autophagy. P, phosphorylation; $\uparrow$, increase; $\downarrow$, decrease.

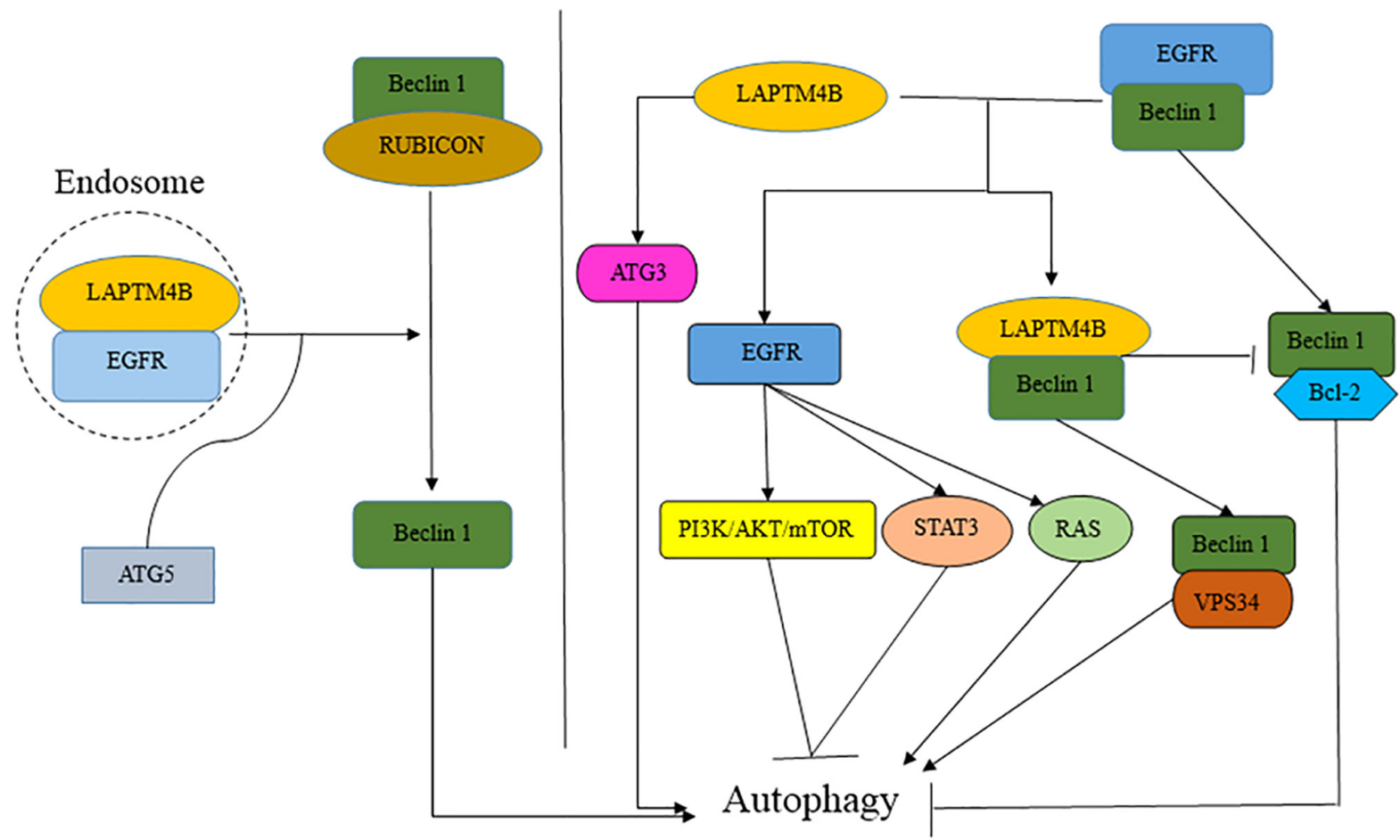

Figure 2. LAPTM4B regulates autophagy through the EGFR signaling pathway.

\section{LAPTM4B and EGFR}

The aforementioned role of EGFR in autophagy is kinase-dependent. It has been reported that inactive EGFR colocalized with LAPTM4B in both late and early endosomes and that these two molecules could interact and stabilize each other in cells grown in the absence of serum (16). As aforementioned, LAPTM4B is required for autophagy initiation. LAPTM4B silencing using siRNA inhibits autophagosome maturation and autophagic flux in the context of metabolic 
stress $(16,18)$. It may be hypothesized that LAPTM4B is involved in autophagy through the EGFR pathway.

LAPTM4B and EGFR are upregulated or mutated in the majority of types of cancer, including lung cancer and breast cancer, and associated with cancer cell proliferation and survival, drug resistance and poor prognosis $(8,19)$. Tan et al (16) reported that inactive EGFR and LAPTM4B interact and stabilize each other at endosomes. However, as an endosomal protein, LAPTM4B could modulate inactive EGFR endosomal accumulation and inhibit EGF-stimulated EGFR lysosomal sorting (16). As aforementioned, EGFR phosphorylation is required for autophagy. A previous study has demonstrated that EGFR-TKIs or neutralizing antibody such as cetuximab induce autophagy and exert cytoprotective roles on cancer cells (71). In addition, EGFR silencing using siRNA strongly inhibits autophagosome formation (16). Notably, it has been demonstrated that LAPTM4B serves a key role in autophagy and EGFR gene mutations in clinical tumor samples $(16,18,72)$. LAPTM4B is required for the endosomal accumulation of inactive EGFR and autophagy, and LAPTM4B is a cofactor for inactive EGFR-driven autophagy (16).

The mechanism of LAPTM4B in EGFR-mediated autophagy is as follows (Fig. 2). Beclin 1, as well as ATG3, -5, -6, $-7,-8,-10$ and -12 are important mediators of autophagy $(2,3)$. On one hand, LAPTM4B interacts and stabilizes with inactive EGFR at endosomes, thus recruiting ATG5 to disassociate Beclin 1 from the RUBICON-Beclin 1 complex to trigger autophagy (16). On the other hand, the expression levels of LAPTM4B positively correlate with EGFR (16). In addition, Tian et al (73) demonstrate that Beclin1 interacted with the $\mathrm{N}$ - and C-terminal domains of LAPTM4B and compete with EGFR for LAPTM4B binding. Nevertheless, this study did not clarify the exact mechanism of the relationship between LAPTM4B and EGFR. Notably, the Beclin 1/EGFR complex can also inhibit autophagy (13). Thus, it may be hypothesized that LAPTM4B competes with EGFR to interact with Beclin1 to antagonize autophagy inhibition (Fig. 2). LAPTM4B may also regulate autophagy through EGFR signaling pathways, including PI3K/AKT/mTOR, EGFR-RAS, EGFR-STAT3.

\section{Conclusions and future perspectives}

LAPTM4B regulates autophagy either by directly activating ATG3 transcription, or through the EGFR pathway (whether active or inactive). The present review aimed to describe the role and mechanism of action of LAPTM4B in EGFR-mediated autophagy. EGFR-TKI therapy for EGFR sensitive mutations induces autophagy contributing to cancer cell survival. Considering the role of LAPTM4B in autophagy and chemotherapy resistance, full understanding of the association between LAPTM4B, EGFR and autophagy may provide insight into more effective therapeutic strategies for tumors such as non-small-cell lung carcinoma. LAPTM4B may be a potential candidate for future tumor treatment options, particularly in combination with other cancer driver gene targeted therapy such as EGFR-TKIs. The present review may provide useful information for future studies on the development of more effective targeted therapies compared with broad spectrum chemotherapy.

\section{Acknowledgements}

Not applicable.

\section{Funding}

No funding was received.

\section{Availability of data and materials}

Data sharing is not applicable to this article, as no data sets were generated or analyzed during the current study.

\section{Authors' contributions}

XJ and HM drafted the manuscript. XJ and YD designed the study, supervised preparation of the manuscript and gave final approval of the version to be published. Data authentication is not applicable. All authors read and approved the final manuscript.

\section{Ethics approval and consent to participate}

Not applicable.

\section{Patient consent for publication}

Not applicable.

\section{Competing interests}

The authors declare that they have no competing interests.

\section{References}

1. Mizushima N, Levine B, Cuervo AM and Klionsky DJ: Autophagy fights disease through cellular self-digestion. Nature 451: 1069-1075, 2008.

2. Klionsky DJ, Abdelmohsen K, Abe A, Abedin MJ, Abeliovich $\mathrm{H}$, Arozena AA, Adachi H, Adams CM, Adams PD, Adeli K, et al: Guidelines for the use and interpretation of assays for monitoring autophagy (3rd edition). Autophagy 12: 1-222, 2016.

3. Feng Y, He D, Yao Z and Klionsky DJ: The machinery of macroautophagy. Cell Res 24: 24-41, 2014.

4. Henson ES and Gibson SB: Surviving cell death through epidermal growth factor (EGF) signal transduction pathways: Implications for cancer therapy. Cell Signal 18: 2089-2097, 2006.

5. Jutten B and Rouschop KMA: EGFR signaling and autophagy dependence for growth, survival, and therapy resistance. Cell Cycle 13: 42-51, 2014.

6. Henson E, Chen Y and Gibson S: EGFR family members' regulation of autophagy is at a crossroads of cell survival and death in cancer. Cancers (Basel) 9: 27, 2017.

7. Sigismund S, Avanzato D and Lanzetti L: Emerging functions of the EGFR in cancer. Mol Oncol 12: 3-20, 2018.

8. Mendelsohn X and Baselga J: Epidermal growth factor receptor targeting in cancer. Semin Oncol 33: 369-385, 2006.

9. Paez JG, Jänne PA, Lee JC, Tracy S, Greulich H, Gabriel S, Herman P, Kaye KJ, Lindeman N, Boggon TJ, et al: EGFR mutations in lung cancer: Correlation with clinical response to gefitinib therapy. Science 304: 1497-1500, 2004.

10. Majem M and Remon J: Tumor heterogeneity: Evolution through space and time in EGFR mutant non small cell lung cancer patients. Transl Lung Cancer Res 2: 226-237, 2013.

11. Feng Y, Gao S, Gao Y, Wang X and Chen Z: Anti-EGFR antibody sensitizes colorectal cancer stem-like cells to Fluorouracil-induced apoptosis by affecting autophagy. Oncotarget 7: 81402-81409, 2016. 
12. Han W, Pan H, Chen Y, Sun J, Wang Y, Li J, Ge W, Feng L, Lin X, Wang X, et al: EGFR tyrosine kinase inhibitors activate autophagy as a cytoprotective response in human lung cancer cells. PLoS One 6: e18691, 2011.

13. Wei Y, Zou Z, Becker N, Anderson M, Sumpter R, Xiao G Kinch L, Koduru P, Christudass CS, Veltri RW, et al: EGFR-mediated phosphorylation of beclin 1 in autophagy suppression, tumor progression and tumor chemoresistance. Cell 154: 1269-1284, 2013

14. Liu XR, Zhou RL, Zhang QY, Zhang Y, Jin YY, Lin M, Rui JA and Ye DX: Structure analysis and expressions of a novel tetratransmembrane protein, lysosoma-associated protein transmembrane 4 beta associated with hepatocellular carcinoma. World J Gastroenterol 10: 1555-1559, 2004

15. Tooze SA and Yoshimori T: The origin of the autophagosomal membrane. Nat Cell Biol 12: 831-835, 2010.

16. Tan X, Thapa N, Sun Y and Anderson RA: A kinase independent role for EGF receptor in autophagy initiation. Cell 160: 145-160, 2015.

17. Wang F, Wu H, Zhang S, Lu J, Lu Y, Zhan P, Fang Q, Wang F Zhang $\mathrm{X}$, Xie $\mathrm{C}$ and Yin Z: LAPTM4B facilitates tumor growth and induces autophagy in hepatocellular carcinoma. Cancer Manag Res 11: 2485-2497, 2019.

18. Li Y, Zhang Q, Tian R, Wang Q, Zhao JJ, Iglehart JD, Wang ZC and Richardson AL: Lysosomal transmembrane protein LAPTM4B promotes autophagy and tolerance to metabolic stress in cancer cells. Cancer Res 71: 7481-7489, 2011

19. Meng Y, Wang L, Chen D, Chang Y, Zhang M, XU JJ, Zhou R and Zhang QY: LAPTM4B: An oncogene in various solid tumors and its functions. Oncogene 35: 6359-6365, 2016.

20. Wang S, Zhang QY and Zhou RL: Relationship between LAPTM4B gene polymorphism and susceptibility of primary liver cancer. Ann Oncol 23: 1864-1869, 2012

21. Wang B, Xu JJ,Zhou R and Zhang QY: Association of LAPTM4B gene polymorphism with nasopharyngeal carcinoma susceptibility in a Chinese population. Med Oncol 30: 470, 2013.

22. Tang H, Tian H, Yue W, Li L, Li S, Gao C, Si L, Qi L, Lu M and $\mathrm{Hu}$ W: LAPTM4B polymorphism is associated with nonsmall cell lung cancer susceptibility and prognosis. Oncol Rep 31: 2454-2460, 2014.

23. Shaker O, Taha F, Salah M and El-Marzouky M: LAPTM4B gene expression and polymorphism as diagnostic markers of breast cancer in Egyptian patients. J Med Biochem 34: 393-401, 2015.

24. Xia LZ, Yin ZH, Ren YW, Shen L, Wu W, Li XL, Guan P and Zhou BS: The relationship between LAPTM4B polymorphisms and cancer risk in Chinese Han population: A meta-analysis. Springerplus 4: 179, 2015

25. Yang H, Xiong F, Wei X, Yang Y, McNutt MA and Zhou RL: Overexpression of LAPTM4B-35 promotes growth and metastasis of hepatocellular carcinoma in vitro and in vivo. Cancer Lett 294: 236-244, 2010.

26. Liu X, Xiong F, Wei X, Yang H and Zhou R: LAPTM4B-35, a novel tetratransmembrane protein and its PPRP motif serve critical roles in proliferation and metastatic potential of hepatocellular carcinoma cells. Cancer Sci 100: 2335-2340, 2009.

27. Li L, Wei XH, Pan YP, Li HC, Yang H, He QH, Pang Y, Shan Y, Xiong FX, Shao GZ and Zhou RL: LAPTM4B: A nove cancer-associated gene motivates multidrug resistance through efflux and activating PI3K/AKT signaling. Oncogene 29: 5785-5795, 2010.

28. Li Y, Zou L, Li Q, Haibe-Kains B, Tian R, Li Y, Desmedt C, Sotiriou C, Szallasi Z, Iglehart JD, et al: Amplification of LAPTM4B and YWHAZ contributes to chemotherapy resistance and recurrence of breast cancer. Nat Med 6: 214-218 2010.

29. Zhou L, He XD, Yu JC, Zhou RL, Yang H, Qu Q and Rui JA: Overexpression of LAPTM4B promotes growth of gallbladder carcinoma cells in vitro. Am J Surg 199: 515-521, 2010.

30. Zhou L, He XD, Cui QC, Zhou WX, Qu Q, Zhou RL, Rui JA and Yu JC: Expression of LAPTM4B-35: A novel marker of progression, invasiveness and poor prognosis of extrahepatic cholangiocarcinoma. Cancer Lett 264: 209-217, 2008.

31. Xu Y, Liu Y, Zhou R, Meng F, Gao Y, Yang S, Li X, Yang M and Lou G: LAPTM4B polymorphisms is associated with ovarian cancer susceptibility and its prognosis. Jpn J Clin Oncol 42: 413-419, 2012

32. Yang Y, Yang H, McNutt MA, Xiong F, Xiu N, Li L and Zhou R: LAPTM4B overexpression is an independent prognostic marker in ovarian carcinoma. Oncol Rep 20: 1077-1083, 2008.
33. Zhang H, Tian B, Yu H, Yao H and Gao Z: LAPTM4B-35 protein as a potential therapeutic target in gastric cancer. Tumour Biol 35: 12737-12742, 2014

34. Cheng XJ, Xu W, Zhang QY and Zhou RL: Relationship between LAPTM4B gene polymorphism and susceptibility of colorectal and esophageal cancers. Ann Oncol 19: 527-532, 2008.

35. Usman RM, Razzaq F, Akbar A, Farooqui AA, Iftikhar A, Latif A, Hassan H, Zhao J, Carew JS, Nawrocki ST and Anwer F: Role and mechanism of autophagy-regulating factors in tumorigenesis and drug resistance. Asia Pac J Clin Oncol 17: 193-208, 2021

36. Scarlatti F, Granata R, Meijer AJ and Codogno P: Does autophagy have a license to kill mammalian cells? Cell Death Differ 16: 12-20, 2009.

37. Eisenberg-Lerner A, Bialik S, Simon HU and Kimchi A: Life and death partners: Apoptosis, autophagy and the cross-talk between them. Cell Death Differ 16: 966-975, 2009.

38. Huang J, Ni J, Liu K, Yu Y, Xie M, Kang R, Vernon P, Cao L and Tang D: HMGB1 promotes drug resistance in osteosarcoma. Cancer Res 72: 230-238, 2012.

39. Pan B, Chen D, Huang J, Wang R, Feng B, Song H and Chen L: HMGB1-mediated autophagy promotes docetaxel resistance in human lung adenocarcinoma. Mol Cancer 13: 165, 2014.

40. Tao H, Chen F, Liu H, Hu Y, Wang Y and Li H: Wnt/ $\beta$-catenin signaling pathway activation reverses gemcitabine resistance by attenuating beclin1-mediated autophagy in the MG63 human osteosarcoma cell line. Mol Med Rep 16: 1701-1706, 2017.

41. Ying H, Qu D, Liu C, Ying T, Lv J,Jin S and Xu H: Chemoresistance is associated with Beclin-1 and PTEN expression in epithelial ovarian cancers. Oncol Lett 9: 1759-1763, 2015.

42. Liu Y, Zhao L, Ju Y, Li W, Zhang M, Jiao Y, Zhang J, Wang S, Wang Y, Zhao M, et al: A novel androstenedione derivative induces ROS-mediated autophagy and attenuates drug resistance in osteosarcoma by inhibiting macrophage migration inhibitory factor (MIF). Cell Death Dis 5: e1361, 2014.

43. Eum KH and Lee M: Targeting the autophagy pathway using ectopic expression of beclin 1 in combination with rapamycin in drug-resistant v-Ha-ras-transformed NIH 3T3 cells. Mol Cells 31: 231-238, 2011

44. Wu MY, Fu J, Xu J, O'Malley BW and Wu RC: Steroid receptor coactivator 3 regulates autophagy in breast cancer cells through macrophage migration inhibitory factor. Cell Res 22: 1003-1021, 2012.

45. Pietrocola F, Pol J, Vacchelli E, Baracco EE,Levesque S, Castoldi F, Maiuri MC, Madeo F and Kroemer G: Autophagy induction for the treatment of cancer. Autophagy 12: 1962-1964, 2016.

46. Chude CI and Amaravadi RK: Targeting autophagy in cancer: Update on clinical trials and novel inhibitors. Int J Mol Sci 18: 1279, 2017.

47. Pellegrini P, Strambi A, Zipoli C, Hägg-Olofsson M, Buoncervello M, Linder S and Milito AD: Acidic extracellular $\mathrm{pH}$ neutralizes the autophagy-inhibiting activity of chloroquine: Implications for cancer therapies. Autophagy 10: 562-571, 2014.

48. Fung C, Chen X, Grandis JR and Duvvuri U: EGFR tyrosine kinase inhibition induces autophagy in cancer cells. Cancer Biol Ther 13: 1417-1424, 2012

49. Gorzalczany Y, Gilad Y, Amihai D, Hammel I, Sagi-Eisenberg R and Merimsky O: Combining an EGFR directed tyrosine kinase inhibitor with autophagy-inducing drugs: A beneficial strategy to combat non-small cell lung cancer. Cancer Lett 310: 207-215, 2011.

50. Pan B, Chen Y, Song H, Xu Y, Wang R and Chen L: Mir-24-3p downregulation contributes to VP16-DDP resistance in small-cell lung cancer by targeting ATG4A. Oncotarget 6: 317-331, 2015.

51. Zhu J, Li Y, Tian Z, Hua X, Gu J, Li J, Liu C, Jin H, Wang Y, Jiang G, et al: ATG7 overexpression is crucial for tumorigenic growth of bladder cancer in vitro and in vivo by targeting the ETS2/miRNA196b/FOXO1/p27 axis. Mol Ther Nucleic Acids 7: 299-313, 2017.

52. Chen J, Zhang L, Zhou H, Wang W, Luo Y, Yang $\mathrm{H}$ and $\mathrm{Yi} H$ : Inhibition of autophagy promotes cisplatin-induced apoptotic cell death through Atg5 and beclin 1 in A549 human lung cancer cells. Mol Med Rep 17: 6859-6865, 2018.

53. Wu J, Li W, Ning J, Yu W, Rao T and Cheng F: Long noncoding RNA UCA1 targets miR-582-5p and contributes to the progression and drug resistance of bladder cancer cells through ATG7-mediated autophagy inhibition. Onco Targets Ther 12: 495-508, 2019.

54. Wu Z, Cai L, Lu J, Wang CD, Guan J, Chen X, Wu J, Zheng W, Wu Z, Li Q and Su Z: MicroRNA-93 mediates cabergolineresistance by targeting ATG7 in prolactinoma. J Endocrinol: Sep 1, 2018 (Epub ahead of print). 
55. Lemmon MA and Schlessinger J: Cell signaling by receptor tyrosine kinases. Cell 141: 1117-1134, 2010.

56. Botti J, Djavaheri-Mergny M, Pilatte $\mathrm{Y}$ and Codogno P: Autophagy signaling and the cogwheels of cancer. Autophagy 2: 67-73, 2006.

57. Kim MJ, Woo SJ, Yoon CH, Lee JS, An S, Choi YH, Hwang SG, Yoon G and Lee SJ: Involvement of autophagy in oncogenic K-ras-induced malignant cell transformation. J Biol Chem 286: 12924-12932, 2011.

58. Elgendy M, Sheridan C, Brumatti G and Martin SJ: Oncogenic ras-induced expression of noxa and beclin-1 promotes autophagic cell death and limits clonogenic survival. Mol Cell 42: 23-35, 2011.

59. Ge J, Liu Y, Li Q, Guo X, Gu L, Ma ZG and Zhu YP: Resveratrol induces apoptosis and autophagy in T-cell acute lymphoblastic leukemia cells by inhibiting Akt/mTOR and activating p38-MAPK. Biomed Environ Sci 26: 902-911, 2013.

60. Alers S, Löffler AS, Wesselborg S and Stork B: Role of AMPK-mTOR-Ulk1/2 in the regulation of autophagy: Cross talk, shortcuts, and feedbacks. Mol Cell Biol 32: 2-11, 2012.

61. Kim J, Kundu M, Viollet B and Guan KL: AMPK and mTOR regulate autophagy through direct phosphorylation of Ulk1. Nat Cell Biol 13: 132-141, 2011.

62. Schmukler E, Kloog Y and Pinkas-Kramarski R: Ras and autophagy in cancer development and therapy. Oncotarget 5: 577-586, 2014.

63. Kim YM, Jung CH, Seo M, Kim EK, Park JM, Bae SS and Kim DH: mTORC1 phosphorylates UVRAG to negatively regulate autophagosome and endosome maturation. Mol Cell 57: 207-218, 2015.

64. Wu SY, Lan SH, Cheng DE, Chen WK, Shen CH, Lee YR Zuchini R and Liu HS: Ras-related tumorigenesis is suppressed by BNIP3-mediated autophagy through inhibition of cell proliferation. Neoplasia 13: 1171-1182, 2011.
65. Byun JY, Yoon CH, An S, Park IC, Kang CM, Kim MJ and Lee SJ: The Rac1/MKK7/JNK pathway signals upregulation of Atg5 and subsequent autophagic cell death in response to oncogenic ras. Carcinogenesis 30: 1880-1888, 2009.

66. Liu D, Lin J, Su J, Chen X, Jiang P and Huang K: Glutamine deficiency promotes PCV2 infection through induction of autophagy via activation of ROS-mediated JAK2/STAT3 signaling pathway. J Agric Food Chem 66: 11757-11766, 2018.

67. Quesnelle KM, Boehm AL and Grandis JR: STAT-mediated EGFR signaling in cancer. J Cell Biochem 102: 311-319, 2007.

68. Pattingre S, Tassa A, Qu X, Garuti R, Liang XH, Mizushima N, Packer M, Schneider MD and Levine B: Bcl-2 antiapoptotic proteins inhibit beclin 1-dependent autophagy. Cell 122: 927-939, 2005.

69. Rouschop KMA, van den Beucken T, Dubois L, Niessen H, Bussink J, Savelkouls K, Keulers T, Mujcic H, Landuyt W, Voncken JW, et al: The unfolded protein response protects human tumor cells during hypoxia through regulation of the autophagy genes MAP1LC3B and ATG5. J Clin Invest 120: 127-141, 2010.

70. Shen S, Niso-Santano M, Adjemian S, Takehara T, Malik SA Minoux H, Souquere S, Mariño G, Lachkar S, Senovilla L, et al: Cytoplasmic STAT3 represses autophagy by inhibiting PKR activity. Mol Cell 48: 667-680, 2012.

71. Eimer S, Belaud-Rotureau MA, Airiau K, Jeanneteau M, Laharanne E, Véron N, Vital A, Loiseau H, Merlio JP and Belloc F: Autophagy inhibition cooperates with erlotinib to induce glioblastoma cell death. Cancer Biol Ther 11: 1017-1027, 2011.

72. Wang L, Meng Y and Zhang QZ: LAPTM4B is a novel diagnostic and prognostic marker for lung adenocarcinoma and associated with mutant EGFR. BMC Cancer 19: 293, 2019.

73. Tian M, Chen Y, Tian D, Qiao X, Ma Z and Li J: Beclin1 antagonizes LAPTM4B-mediated EGFR overactivation in gastric cancer cells. Gene 626: 48-53, 2017. 